\title{
Elementary excitations in anisotropic nanofilms of multiferroics with competing interactions at the interface
}

\author{
I. F. Sharafullin ${ }^{\dagger, 1}$, H. T. Diep ${ }^{2}$ \\ †SharafullinIF@yandex.ru \\ ${ }^{1}$ Bashkir State University, 32 Zaki Validi St., Ufa, 450076, Russia \\ ${ }^{2}$ Laboratory of Theoretical Physics and Modeling, CNRS UMR 8089, Cergy Paris University, 2 Avenue Adolphe Chauvin, \\ Cergy-Pontoise, 95302, France
}

In this work we study, using the of two-time Green's functions method, the spectrum of spin waves in a monolayer and bilayer of anisotropic magnetic film sandwiched between the ferroelectric layers in a magnetoelectric superlattice. Surface spin configuration is calculated by minimizing the interaction energy. It is shown that the angles between spins near the surface are strongly modified with respect to the bulk configuration. We include the anisotropy energy between the spin vectors at sites $i$ and $j$ in the Hamiltonian of multiferroic superlattice. The anisotropy energy stabilizes the angle between local quantization axes. The anisotropy parameter $K_{i, j}$ supposed positive, and nonzero only for the nearest interacting neighbors. It is shown that the magnetoelectric interaction strongly affects the long-wavelength mode of spin waves. The magnetization of the magnetic film at low temperatures was also calculated. The magnetization $M$ is strongly dependent on temperatures: at higher $T$, the larger the magnetoelectric interaction, the greater is $M$. However, at $T=0$, the spin length becomes smaller for large values of the magnetoelectric interaction due to the so-called spin reduction that takes place in antiferromagnets. As a result, the crossover (intersection) of the magnetization curves occurs for different values of $\theta$ at low $T$.

Keywords: superlattice, Green's functions, spectrum of spin waves, magnetoelectric interaction.

УДК: 538.9

\section{Элементарные возбуждения в анизотропных нанопленках мультиферроиков с конкурирующими поверхностными взаимодействиями}

\author{
Шарафулин И. Ф. ${ }^{\dagger, 1}$, Диеп Х. ${ }^{2}$ \\ ${ }^{1}$ Башкирский государственный университет, ул. Заки Валиди, 32, Уфа, 450076, Россия \\ ${ }^{2}$ Лаборатория теоретической физики и моделирования университета Сержи-Париж, ЦНРС, УМР 8089 , \\ Авеню Адольфа Шавина, 2, Сержи-Понтуаз, 95302, Франция
}

Целью работы является исследование элементарных возбуждений и влияния магнито-электрической связи на спектр спиновых волн в анизотропных нанопленках мультиферроиков с конкурирующими поверхностными взаимодействиями, а также исследование спиновой конфигурации основного состояния поверхностных слоев магнитной пленки с конкурирующими обменным, магнитоэлектрическим взаимодействиями и внешним магнитным полем, действующим перпендикулярно плоскости пленок сверхрешетки. Используя метод двухвременных температурных функций Грина, исследуется спектр спиновых волн в однослойной и двуслойной анизотропной магнитной пленке, находящейся между сегнетоэлектрическими слоями магнитоэлектрической сверхрешетки. В гамильтониане учтены энергии обменного ферромагнитного взаимодействия и энергия анизотропии между векторами спинов на узлах $i$ и $j$, которая стабилизирует угол между их локальными осями квантования в магнитной пленке, энергия диполь-дипольного взаимодействия в сегнетоэлектрической пленке, a также энергия магнитоэлектрического взаимодействия на интерфейсе пленок. Параметр анизотропии $K_{i, j}$ предполагаем положительным, и отличным от нуля только для ближайших взаимодействующих соседей. Показано, что магнитоэлектрическое взаимодействие оказывает сильное воздействие на длинноволновую моду спиновых 
волн. Рассчитана также намагниченность магнитной пленки при низких температурах. Намагниченность $M$ сильно зависит от температур: при высоких $T$ чем больше магнитоэлектрическое взаимодействие тем больше $M$. Однако при $T=0$ уменьшается модуль намагниченности при возрастании магнитоэлектрического взаимодействия из-за эффекта спинового сокращения. Такой эффект имеет место в антиферромагнетиках. Как следствие, происходит кроссовер (пересечение) кривых намагниченности для различных значений $\theta$ при низкой $T$.

Ключевые слова: сверхрешетка, функции Грина, элементарные возбуждения, спектр спиновых волн, магнитоэлектрическое взаимодействие.

\section{Introduction}

Non-collinear spin structures, which are quite interesting by themselves, became the subject of attention after the discovery of electrical polarization in some of them [1,2]. The existence of polarization is possible due to the inhomogeneous magnetoelectric effect, namely that electrical polarization can occur in the domain of magnetic heterogeneity. In non collinear structures, the microscopic mechanism of the coupling of polarization and the relative orientation of the magnetization vectors is based on the interaction of Dzyaloshinskii-Moriya [2-5]. Surface ME effects appears due to the charge and spin accumulation [6-7]. Thus, the Dzyaloshinskii-Moriya interaction connects the angle between the spins and the magnitude of the displacement of non-magnetic ions. In some micromagnetic structures all ligands are shifted in one direction, which leads to the appearance of macroscopic electrical polarization. In [8] it was shown that the most extensive class of candidates for the detection of magnetic vortices and anti-vortices includes the interfaces of magnetic and magnetoelectric superlattices. In such type of structures the geometry of the material breaks the central symmetry and, therefore, can lead to the appearance of chiral magnetoelectric interactions. Note here that properties of a helimagnetic thin film with quantum Heisenberg spin model by using the Green's function method was investigated in [9]. Surface spin configuration is calculated by minimizing the spin interaction energy. The transition temperature is shown to depend strongly on the helical angle. Results are in agreement with existing experimental observations on the stability of helical structure in thin films and on the insensitivity of the transition temperature with the film thickness.

Recent studies are focused on the interface-induced effects of magnetoelectric superlattices [10-16]. The superstructures naturally lead to the interaction of magnetic vortices on different interfaces, which has unique dynamics compared to the interaction of the vortices in the 2D magnetic films.

We consider in this paper a magnetic films sandwiched between ferroelectric films. The aim of this paper is to propose a new model for the coupling between the magnetic film and the ferroelectric film by introducing a chiral magnetoelectric interaction. It turns out that this interface coupling gives rise to non collinear spin configurations in zero applied magnetic field. Using the Green's function method, we study spinwave excitations in zero field of a two type of superlattices: anisotropic magnetic monolayer sandwiched between monolayer ferroelectric film and anisotropic magnetic bilayer films sandwiched between two ferroelectric layers. We find that the chiral magnetoelectric interaction affects strongly the long wave-length mode.
The Chapter is organized as follows. Section 2 is devoted to the description of our model and the determination of the ground-state spin configuration. In section 3 we show the results of the Green's function technique in zero field for a monolayer and a bilayer. Concluding remarks are given in section 4 .

\section{Model and ground state}

The Hamiltonian of this multiferroic superlattice is expressed as:

$$
\begin{aligned}
H= & -\sum_{i, j} J_{i j}^{m} \vec{S}_{i} \cdot \vec{S}_{j}-\sum_{i, j} J_{i j}^{f} \vec{P}_{i} \cdot \vec{P}_{j}-\sum_{i, j, k} J^{m f} e_{i, j} \vec{P}_{k} \cdot\left[\vec{S}_{i} \times \vec{S}_{j}\right]- \\
& -\sum_{\langle i, j\rangle} K_{i, j} S_{i}^{z} S_{j}^{z} \cos \theta_{i, j}
\end{aligned}
$$

where $\vec{S}_{i}$ is the spin on the $i$-th site, $J_{i j}^{m}>0$ the ferromagnetic interaction parameter between a spin and its nearest neighbors (NN) and the sum is taken over $\mathrm{NN}$ spin pairs. We consider $J_{i j}^{m}>0$ to be the same, namely $J^{m}$, for spins everywhere in the magnetic film.

We describe the magnetic film with the Heisenberg spin model on a simple cubic lattice. $\vec{P}_{i}$ is the polarization on the $i$-th lattice site, polarizations are Ising-like vectors of magnitude 1, pointing in the $\pm z$ direction, $J_{i j}^{f}$ the interaction parameter between $\mathrm{NN}$ and the sum is taken over $\mathrm{NN}$ sites. Similar to the ferromagnetic subsystem we will take the same $J_{i j}^{f}=J^{f}$ for all ferroelectric sites. The coefficient of the interface coupling is proportional to $\left\langle P_{k}\right\rangle$ which depends on $T$. In last ansotropic term $K_{i, j}$ is supposed to be positive and we take $K_{i, j}=K$ for NN pair in the $x y$ plane, for simplicity. If we choose the vector $J^{m f} e_{i, j} \vec{P}_{k}$ perpendicular to the $x y$ plane then the interface interaction energy is minimum when the spins are in the $x y$ plane because $J^{m f} e_{i, j} \vec{P}_{k}$ is parallel to $\left[\vec{S}_{i} \times \vec{S}_{j}\right]$. One can choose any orientation for $J^{m f} e_{i, j} \vec{P}_{k}$ but in that case to have the minimum energy the plane containing $\vec{S}_{i}$ and $\vec{S}_{j}$ should be perpendicular to $J^{m f} e_{i, j} \vec{P}_{k}$ : the spins are not in the $x y$ plane, making the spin ground state configuration analysis difficult.

In nanofilms of superlattices the magnetoelectric interaction is crucial for the creation of non-collinear longrange spin ordering.

Let us determine the ground state spin configurations in magnetic layers, sandwiched between ferroelectric layer. Since the polarizations are along the $z$ axis, the interface magnetoelectric interaction is minimum when $\overrightarrow{\boldsymbol{S}}_{i}$ and $\overrightarrow{\boldsymbol{S}}_{j}$ lie in the $x y$ interface plane and perpendicular to each other. However the ferromagnetic exchange interaction among the spins will compete with the perpendicular $\vec{P}_{k} \cdot\left[\vec{S}_{i} \times \vec{S}_{j}\right]$ configuration. The resulting configuration is non collinear. We note that the ferroelectric film has always polarizations along the $z$ axis even when interface interaction is turned on. 
By symmetry, each spin has the same angle $\theta$ with its four nearest neighbors in the $x y$ plane. The energy of the spin $\overrightarrow{\boldsymbol{S}}_{i}$ gives the relation between $\theta$ and $J^{m}$

$$
E_{i}=-4 J^{m} S^{2} \cos \theta+8 J^{m f} P^{z} S^{2} \sin \theta,
$$

where $\theta=\left|\theta_{i, j}\right|$ and care has been taken on the signs of $\sin \theta_{i, j}$ when counting NN, namely two opposite NN have opposite signs, and the oppossite coefficient $e_{i, j}$. Note that the coefficient 8 of the second term is due to the fact that each spin has 4 coupling spin pairs with the NN polarization in the upper ferroelectric plane, and 4 with the NN polarization of the lower ferroelectric plane (we are in the case of a magnetic monolayer). The minimization of $E_{i}$ yields, taking $P^{z}=1$ in the ground state and $S=1$,

$$
\frac{d E_{i}}{d \theta}=0 \Rightarrow-\frac{2 J^{m f}}{J^{m}}=\tan \theta \Rightarrow \theta=\arctan \left(-\frac{2 J^{m f}}{J^{m}}\right) .
$$

The value of $\theta$ for a given $-2 J^{m f} / J^{m}$. We see that when $J^{m f} \rightarrow 0$, one has $\theta \rightarrow 0$, and when $J^{m f} \rightarrow-\infty$, one has $\theta \rightarrow \pi / 2$ as it should be. Note that we will consider in this paper $J^{m f}<0$ so as to have $\theta>0$.

In the case when the magnetic film has a thickness, the angle between nearest neighbors spins in each magnetic layer is different from that of the neighboring layer. It is more convenient using the numerical minimization method called "steepest descent method" to obtain the GS spin configuration. We use a sample size $N \times N \times L$. For most calculations, we select $N=40$ and $L=8$ using the periodic boundary conditions in the $x y$ plane. Exchange parameters between spins and polarizations are taken as $J^{m}=J^{f}=1$.

We investigated the following range of values for the interaction parameters $J^{m f}$ : from $J^{m f}=0$ to $J^{m f}=-6.0$ with different values of the external magnetic and electric fields. Fig. 1 shows the GS configurations of the magnetic interface layer for small values of $J^{m f}:-0.15,-0.75$. Such small values yields small values of angles between spins so that the ground state configurations have ferromagnetic and non collinear domains.

For larger values of $J^{m f}$, the GS spin configurations have periodic structures with no more mixed domains. We show in Fig. S1a (Supplementary Material) examples where $J^{m f}=-0.15$ and -0.75 . One can see that each spin has the same turning angle $\theta$ with its NN in both $x$ and $y$ direction. This explains the structures shown in Figs. S1b. The periodicity of the diagonal parallel lines depends on the value of $\theta$. With a large size of $N$, the periodic conditions have no significant effects.

\section{Spin waves in zero field}

Let us show theoretically spin-waves (SW) excited in the magnetic film in zero field. The method we employ is the Green's function technique for non- collinear spin configurations. In this section, we consider the same Hamiltonian supposed in Eq. (1) but with quantum spins of amplitude $1 / 2$.

As seen in the previous section, the spins lie in the $x y$ planes, each on its quantization local axis lying in the

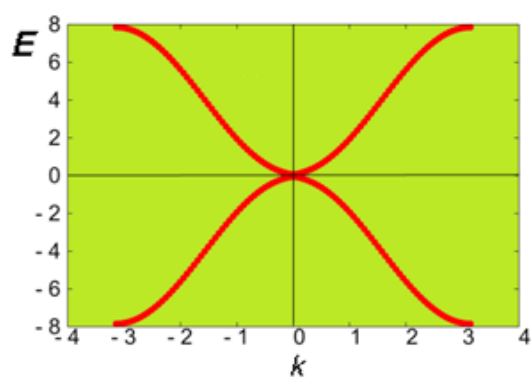

a

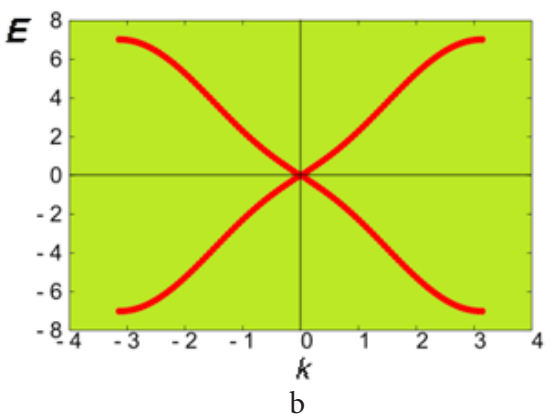

Fig. 1. Spin-wave energy $E(k)$ versus $k\left(k=k_{x}=k_{z}\right)$ for $\theta=0.3$ radian (a) and $\theta=1$ (b) in $2 \mathrm{D}$ at $T=0$. See text for comments.

$x y$ plane. Expressing the spins in the local coordinates, one has

$$
\begin{array}{r}
\overrightarrow{\boldsymbol{S}}_{i}=S_{i}^{x} \hat{x}_{i}+S_{i}^{y} \hat{y}_{i}+S_{i}^{z_{i}} \hat{z}_{i}, \\
\overrightarrow{\boldsymbol{S}}_{j}=S_{j}^{x_{j}} \hat{x}_{j}+S_{j}^{y_{j}} \hat{y}_{j}+S_{j}^{z_{j}} \hat{z}_{j},
\end{array}
$$

where the $i$ and $j$ coordinates are connected by the rotation

$$
\begin{gathered}
\hat{x}_{j}=\cos \theta_{i, j} \hat{z}_{i}+\sin \theta_{i, j} \hat{x}_{i}, \\
\hat{z}_{j}=-\sin \theta_{i, j} \hat{z}_{i}+\cos \theta_{i, j} \hat{x}_{i}, \quad \hat{y}_{j}=\hat{y}_{i},
\end{gathered}
$$

where $\theta_{i, j}=\theta_{i}-\theta_{j}$ being the angle between $\overrightarrow{\boldsymbol{S}}_{i}$ and $\overrightarrow{\boldsymbol{S}}_{j}$.

The ground state spin configuration for one monolayer is periodically non collinear. For two-layer magnetic film, the spin configurations in two layers are identical by symmetry.

For quantum calculations we consider the case of spin one-half $S=1 / 2$. Expressing the total magnetic Hamiltonian

$$
H=-\sum_{i, i} J_{i j}^{m} \vec{S}_{i} \cdot \vec{S}_{j}-\sum_{i, j, k} J^{m f} e_{i, j} \vec{P}_{k} \cdot\left[\vec{S}_{i} \times \vec{S}_{j}\right]
$$

in the local coordinates. Writing $\overrightarrow{\boldsymbol{S}}_{j}$ in the coordinates $\left(\hat{x}_{i}, \hat{y}_{i}, \hat{z}_{i}\right)$, one gets the following exchange Hamiltonian from Eqs. (1) - (4)

$$
\begin{aligned}
H_{M} & =-\sum_{i, j} J^{m}\left\{\frac{1}{4}\left(\cos \theta_{i, j}-1\right)\left(S_{i}^{+} S_{j}^{+}+S_{i}^{-} S_{j}^{-}\right)\right. \\
& +\frac{1}{4}\left(\cos \theta_{i, j}+1\right)\left(S_{i}^{+} S_{j}^{-}+S_{i}^{-} S_{j}^{+}\right) \\
& +\frac{1}{2} \sin \theta_{i, j}\left(S_{i}^{+}+S_{i}^{-}\right) S_{j}^{z}-\frac{1}{2} \sin \theta_{i, j} S_{i}^{z}\left(S_{j}^{+}+S_{j}^{-}\right) \\
& \left.-\sin \theta_{i, j} S_{i}^{z} S_{j}^{z}\right\} \\
& +\frac{J^{m f} P^{z}}{4} \sum_{\langle i, j\rangle}\left[\left(S_{i}^{+}+S_{i}^{-}\right)\left(S_{j}^{+}+S_{j}^{-}\right)\left|\sin \theta_{i, j}\right|\right. \\
& \left.+4 S_{i}^{z} S_{j}^{z}\left|\sin \theta_{i, j}\right|\right] .
\end{aligned}
$$

Note that $P^{z}=1$ in the ground state. 
We now define the following two double-time Green's functions as

$$
\begin{aligned}
G_{i, j}\left(t, t^{\prime}\right) & =\left\langle\left\langle S_{i}^{+}(t) ; S_{j}^{-}\left(t^{\prime}\right)\right\rangle\right\rangle \\
& =-i \theta\left(t-t^{\prime}\right)\left\langle\left[S_{i}^{+}(t), S_{j}^{-}\left(t^{\prime}\right)\right]\right\rangle, \\
F_{i, j}\left(t, t^{\prime}\right) & =\left\langle\left\langle S_{i}^{-}(t) ; S_{j}^{-}\left(t^{\prime}\right)\right\rangle\right\rangle \\
& =-i \theta\left(t-t^{\prime}\right)\left\langle\left[S_{i}^{-}(t), S_{j}^{-}\left(t^{\prime}\right)\right]\right\rangle .
\end{aligned}
$$

The equations of motion of these functions

$$
\begin{aligned}
i \hbar \frac{d G_{i, j}\left(t, t^{\prime}\right)}{d t} & =\left\langle\left[S_{i}^{+}(t), S_{j}^{-}\left(t^{\prime}\right)\right]\right\rangle \delta\left(t-t^{\prime}\right) \\
& -\left\langle\left\langle\left[H_{M}, S_{i}^{+}\right] ; S_{j}^{-}\right\rangle\right\rangle, \\
i \hbar \frac{d F_{i, j}\left(t, t^{\prime}\right)}{d t} & =\left\langle\left[S_{i}^{-}(t), S_{j}^{-}\left(t^{\prime}\right)\right]\right\rangle \delta\left(t-t^{\prime}\right) \\
& -\left\langle\left\langle\left[H_{M}, S_{i}^{-}\right] ; S_{j}^{-}\right\rangle\right\rangle .
\end{aligned}
$$

For the $H_{m}$ and $H_{a}$ parts, the above equations of motion generate terms such as $\left\langle\left\langle S_{l}^{z} S_{i}^{ \pm} ; S_{j}^{-}\right\rangle\right\rangle$and $\left\langle\left\langle S_{l}^{ \pm} S_{i}^{ \pm} ; S_{j}^{-}\right\rangle\right\rangle$. These functions can be approximated by using the Bogolyubov Tyablikov decoupling [18] to reduce to the above-defined $G$ and $F$ functions:

$$
\begin{aligned}
& \left\langle\left\langle S_{l}^{z} S_{i}^{ \pm} ; S_{j}^{-}\right\rangle\right\rangle \approx\left\langle S_{l}^{z}\right\rangle\left\langle\left\langle S_{i}^{ \pm} ; S_{j}^{-}\right\rangle\right\rangle \\
& \left\langle\left\langle S_{l}^{ \pm} S_{i}^{ \pm} ; S_{j}^{-}\right\rangle\right\rangle \approx\left\langle S_{l}^{ \pm}\right\rangle\left\langle\left\langle S_{i}^{ \pm} ; S_{j}^{-}\right\rangle\right\rangle \approx 0 .
\end{aligned}
$$

For the magnetoelectric term, the commutation relations $\left[H_{m f}, S_{i}^{ \pm}\right]$one can obtain:

$$
J^{m f}\left\langle P^{z}\right\rangle \sum_{l} \sin \theta\left[\mp S_{i}^{z}\left(S_{l}^{+}+S_{l}^{-}\right) \pm 2 S_{i}^{ \pm} S_{l}^{z}\right] .
$$

This leads to the following type of Green's function:

$$
\left\langle\left\langle S_{i}^{z} S_{l}^{ \pm} ; S_{j}^{-}\right\rangle\right\rangle \approx\left\langle S_{i}^{z}\right\rangle\left\langle\left\langle S_{l}^{ \pm} ; S_{j}^{-}\right\rangle\right\rangle
$$

We use the following Fourier transforms in the plane of the and Green's functions:

$$
\begin{aligned}
& G_{i, j}\left(t, t^{\prime}, \omega\right)=\frac{1}{\Delta} \int_{B Z} d \vec{k}_{x y} e^{-i \omega\left(t-t^{\prime}\right)} g\left(\omega, \vec{k}_{x z}\right) e^{i \vec{k}_{x y} \cdot\left(\vec{R}_{i}-\vec{R}_{j}\right)}, \\
& F_{i, j}\left(t, t^{\prime}, \omega\right)=\frac{1}{\Delta} \int_{B Z} d \vec{k}_{x y} e^{-i \omega\left(t-t^{\prime}\right)} f\left(\omega, \vec{k}_{x z}\right) e^{i \vec{k}_{x y} \cdot\left(\vec{R}_{i}-\vec{R}_{j}\right)},
\end{aligned}
$$

where the integral is performed in the first $x y$ Brillouin zone of surface $\Delta$ and $\omega$ is the spin - wave frequency. One can define the energy of spin waves $E$ in the following.

For a monolayer, we obtain after the Fourier transforms

$$
\begin{aligned}
& (E+\Psi) g+\Phi f=2\left\langle S^{z}\right\rangle \\
& -\Phi g+(E-\Psi) f=0
\end{aligned}
$$

where $\Psi$ and $\Phi$ are

$$
\begin{aligned}
\Psi= & -J^{m}\left[8\left\langle S^{z}\right\rangle \cos \theta\left(1+\frac{K}{J^{m}}\right)-4\left\langle S^{z}\right\rangle \gamma(\cos \theta+1)\right] \\
& -4 J^{m f}\left\langle P^{z}\right\rangle \sin \theta\left\langle S^{z}\right\rangle \gamma+8 J^{m f}\left\langle P^{z}\right\rangle \sin \theta\left\langle S^{z}\right\rangle, \\
\Phi= & 4 J^{m}\left\langle S^{z}\right\rangle \gamma(\cos \theta-1)-4 J^{m f}\left\langle P^{z}\right\rangle \sin \theta\left\langle S^{z}\right\rangle \gamma,
\end{aligned}
$$

where $\gamma=\left(\cos k_{x} a+\sin k_{y} a\right) / 2, k_{x}$ and $k_{y}$ being the wavevector components in the planes, $a$ the lattice constant.
The spin waves energies are determined by the equation

$$
\begin{gathered}
(E+\Psi)(E-\Psi)+\Phi^{2}=0 \\
E^{2}-\Psi^{2}+\Phi^{2}=0 \\
E= \pm \sqrt{(\Psi+\Phi)(\Psi-\Phi) .}
\end{gathered}
$$

We see that if $\theta=0$ we have $\Phi$ and the last two terms of $\Psi$ are zero. We recover then the ferromagnetic spin wave dispersion relation

$$
E=2 Z J^{m}\left\langle S^{z}\right\rangle(1-\gamma),
$$

where $Z=4$ is the coordination number of the square lattice (taking $K / J^{m}=0$ ).

On the other hand, if $\theta=\pi$, we can see that $\Psi=8 J^{m}\left\langle S^{z}\right\rangle$ and $\Phi=-8 J^{m}\left\langle S^{z}\right\rangle$. The antiferromagnetic spin wave energy

$$
E=2 Z J^{m}\left\langle S^{z}\right\rangle \sqrt{1-\gamma^{2}}
$$

In the presence of a chiral magnetoelectric interaction, we have $0<\cos \theta<1(0<\theta<\pi / 2)$. If $K / J^{m}=0$, the quantity in the square root of Eq. (6) is always $\geq 0$ for any $\theta$. It is zero at $\gamma=1$. We do not need an anisotropy $K / J^{m}$ to stabilize the spin wave at $T=0$. If $K / J^{m} \neq 0$ then it gives a gap at $\gamma=1$.

We show in Fig. 1 the spin wave energy calculated from Eq. (6) for $\theta=0.3$ radian ( $\cong 17.2$ degrees) and 1 radian ( $\cong 57.30$ degrees). All the figures are obtained with $J^{m}=1$, $J^{f}=1, K=0.001$. The spectrum is symmetric for positive and negative wave vectors and for left and right precessions. Note that for small values of $\theta$ (i. e. small $J^{m f}$ ) $E$ is proportional to $k^{2}$ at low $k$ (Fig. 1), as in ferromagnets.

However, for strong value of magnetoelectric coupling $J^{m f}$ at the interface, energy is proportional to $k$ as seen in Fig. 1b., similar to that in antiferromagnets [13]. The change of behavior is progressive with increasing $J^{m f}$, no sudden transition from $k^{2}$ to behavior is observed.

In the case of $S=1 / 2$, the magnetization is given by

$$
\left\langle S^{z}\right\rangle=\frac{1}{2}-\frac{1}{\Delta} \iint d k_{x} d k_{y}\left[\frac{1}{e^{\frac{E_{i}}{k_{B} T}}-1}+\frac{1}{e^{-\frac{E_{i}}{k_{B} T}}-1}\right],
$$

where for each $\overrightarrow{\boldsymbol{k}}$ one has $\pm E_{i}$ values.

Since the energy depends on the magnetization, $\left\langle S^{z}\right\rangle$ can be calculated at finite temperatures self-consistently using the above formula.

We show in Fig. 2 the magnetization $M\left(\equiv\left\langle S^{z}\right\rangle\right)$ calculated by Eq. (7). $M$ depends strongly on $J^{m f}$ : at high $T$, larger $J^{m f}$ yields stronger $M$. However, at $T=0$ the spin length is smaller for larger $\theta$ due to the so-called spin contraction in antiferromagnets [17]. As a consequence there is a crossover of magnetizations with different $\theta$ at low $T$ as shown in Fig. S2 (Supplementary Material).

Consider magnetic bilayer between two ferroelectric films. By symmetry, spins between the two layers are parallel, the energy of a spin on a layer is

$$
E_{i}=-4 J^{m} S^{2} \cos \theta-J^{m} S^{2}+4 J^{m f} P^{z} S^{2} \sin \theta,
$$

where there are 4 in-plane $\mathrm{NN}$ and one parallel $\mathrm{NN}$ spin on the other layer. The interface coupling is with only one polarization instead of two (see Eq. (2)) for a monolayer for comparison. 
The minimum energy corresponds to $\tan \theta=-J^{m f} / J^{m}$.

Writing the Green's functions for each layer and making Fourier transforms in the $x y$ planes, we obtain a system of coupled equations. For a bilayer, the SW energy is the eigenvalues of the following matrix equation

$$
\boldsymbol{R}(E) \boldsymbol{x}=\boldsymbol{u}
$$

Where

$$
\boldsymbol{x}=\left(\begin{array}{c}
g_{1, n^{\prime}} \\
f_{1, n^{\prime}} \\
g_{2, n^{\prime}} \\
f_{2, n^{\prime}}
\end{array}\right), \quad \boldsymbol{u}=\left(\begin{array}{c}
2\left\langle S_{1}^{z}\right\rangle \delta_{1, n^{\prime}} \\
0 \\
2\left\langle S_{2}^{z}\right\rangle \delta_{2, n^{\prime}} \\
0
\end{array}\right)
$$

where $E=\hbar \omega$ and $\boldsymbol{R}(E)$ is given by

$$
\left(\begin{array}{cccc}
E+\Psi_{1} & \Phi_{1} & \Lambda_{1} & 0 \\
-\Phi_{1} & E-\Psi_{1} & 0 & -\Lambda_{1} \\
\Lambda_{2} & 0 & E+\Psi_{2} & \Phi_{2} \\
0 & -\Lambda_{2} & -\Phi_{2} & E-\Psi_{2}
\end{array}\right)
$$

with

$$
\begin{aligned}
\Psi_{1}= & -J^{m}\left[8\left\langle S_{1}^{z}\right\rangle \cos \theta\left(1+K / J^{m}\right)-4\left\langle S_{1}^{z}\right\rangle \gamma(\cos \theta+1)\right] \\
& -2 J^{m}\left\langle S_{2}^{z}\right\rangle-4 J^{m f}\left\langle P^{z}\right\rangle \sin \theta\left\langle S_{1}^{z}\right\rangle \gamma+8 J^{m f}\left\langle P^{z}\right\rangle \sin \theta\left\langle S_{1}^{z}\right\rangle, \\
\Psi_{2}= & -J^{m}\left[8\left\langle S_{2}^{z}\right\rangle \cos \theta\left(1+K / J^{m}\right)-4\left\langle S_{2}^{z}\right\rangle \gamma(\cos \theta+1)\right] \\
& -2 J^{m}\left\langle S_{1}^{z}\right\rangle-4 J^{m f}\left\langle P^{z}\right\rangle \sin \theta\left\langle S_{2}^{z}\right\rangle+8 J^{m f}\left\langle P^{z}\right\rangle \sin \theta\left\langle S_{2}^{z}\right\rangle, \\
\Phi_{n}= & 4 J^{m}\left\langle S_{n}^{z}\right\rangle \gamma(\cos \theta-1)-4 J^{m f}\left\langle P^{z}\right\rangle \sin \theta\left\langle S_{n}^{z}\right\rangle \gamma, \quad n=1,2 \\
& -2 J^{m}\left\langle S_{1}^{z}\right\rangle-4 J^{m f}\left\langle P^{z}\right\rangle \sin \theta\left\langle S_{2}^{z}\right\rangle+8 J^{m f}\left\langle P^{z}\right\rangle \sin \theta, \\
\Lambda_{n}= & 2 J^{m}\left\langle S_{n}^{z}\right\rangle, \quad n=1,2 .
\end{aligned}
$$

Note that by symmetry, one has $\left\langle S_{1}^{z}\right\rangle=\left\langle S_{2}{ }^{z}\right\rangle$.

We show in Fig. 3 the spin wave spectrum of the bilayer case for a strong value $\theta=0.6$ radian.

The first mode has the $E \propto k$ antiferromagnetic behavior at the long wave-length limit for this strong $\theta$, the higher mode has $E \propto k^{2}$ which is the ferromagnetic wave due to the parallel nearest neighbors spins in the $z$ direction.

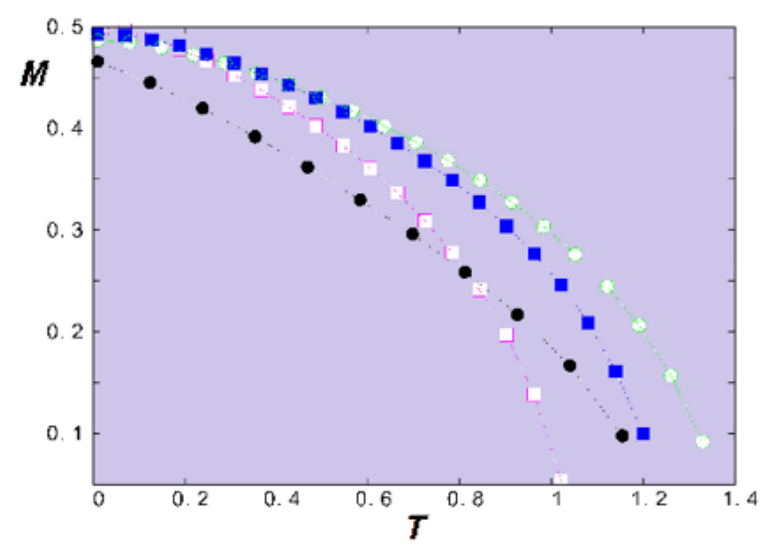

Fig. 2. (Color online) Spin length $M \equiv\left\langle S^{z}\right\rangle$ versus temperature $T$ for a $2 \mathrm{D}$ sheet with $\theta=0.175$ radian (magenta void squares), $\theta=0.524$ (blue filled squares), $\theta=0.698$ (green void circles), $\theta=1.047$ (black filled circles).

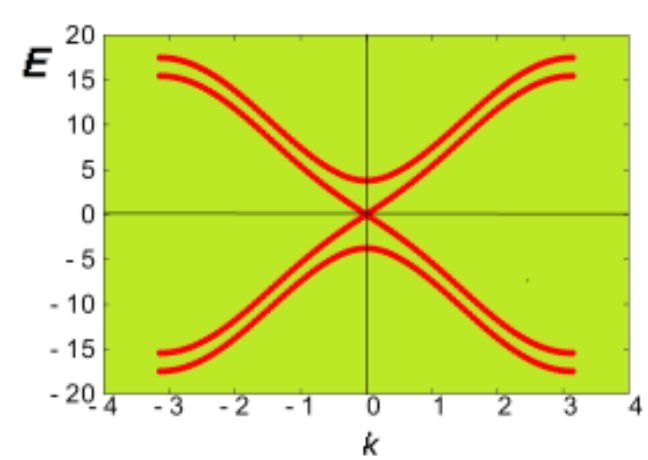

Fig. 3. Spin-wave energy $E$ versus $k=k_{x}=k_{y}$ at $T=0$ for a bilayer with $\theta=0.6$ radian.

\section{Conclusion}

Using the of two-time Green's functions method, we investigate the spin waves energy in a monolayer and bilayer of anisotropic magnetic film sandwiched between the ferroelectric layers in a magnetoelectric superlattice. We include the anisotropy between the spin vectors at sites $i$ and $j$ in the Hamiltonian of multiferroic superlattice. The anisotropy energy stabilizes the angle between local quantization axes. The anisotropy parameter $K_{i, j}$ supposed positive, and nonzero only for the nearest interacting neighbors. In conclusion, we emphasize that the chiral magnetoelectric interaction affects strongly the spin wave mode at $k \rightarrow 0$. Quantum fluctuations in competition with thermal effects cause the cross-over of magnetizations of different $J^{m f}$ : in general stronger $J^{m f}$ yields stronger spin contraction at and near $T=0$ so that the corresponding spin length is shorter. However at higher $T$, stronger $J^{m f}$ means larger $\theta$ which yields stronger magnetization. It explains the cross-over at moderate $T$.

Supplementary Material. The online version of this paper contains supplementary material available free of charge at the journal's Web site (lettersonmaterials.com).

Acknowledgements. S.I. F. acknowledges a financial support - Grant of the Head of the Republic of Bashkortostan 2020.

Author Contributions. Conceptualization, H.T. D. and I.F. S.; methodology, H. T. D.; I. F. S.; writing - original draft preparation, I.F. S.; writing-review and editing, H. T. D. All authors have read and agreed to the published version of the manuscript.

\section{References}

1. S. Dong, X. Zhang, R. Yu, J. M. Liu, E. Dagotto. Physical Review B. 84 (15), 155117 (2011). Crossref

2. M. Mostovoy. Physical Review Letters. 96 (6), 067601 (2006). Crossref

3. H. Katsura, N. Nagaosa, A. V. Balatsky. Physical review letters. 95 (5), 057205 (2005). $\underline{\text { Crossref }}$

4. I. A. Sergienko, E. Dagotto. Physical Review B. 73 (9), 094434 (2006). $\underline{\text { Crossref }}$ 
5. A.P. Pyatakov. Physica B: Condensed Matter. 542, 59 (2018). Crossref

6. A.S. Logginov, G.A. Meshkov, A.V. Nikolaev, E. P. Nikolaeva, A.P. Pyatakov, A.K. Zvezdin. Applied Physics Letters. 93 (18), 182510 (2008). DOI: $10.1063 / 1.3013569$

7. O.G. Udalov, I.S. Beloborodov. AIP Advances. 8 (5), 055810 (2018). $\underline{\text { Crossref }}$

8. A.P. Pyatakov, A. K. Zvezdin, A.M. Vlasov, A.S. Sergeev, D. A. Sechin, E. P. Nikolaeva, L.E. Calvet. Ferroelectrics. 438 (1), 79 (2012). Crossref

9. H.T. Diep. Physical Review B. 91 (1), 014436 (2015). Crossref

10. S. Seki, Y. Onose, Y. Tokura. Phys. Rev. Lett. 101, 067204 (2008). Crossref

11. O. Petrova, O. Tchernyshyov. Phys. Rev. Lett. 84, 214433 (2011).
12. H. B. Braun. Advances in Physics. 61 (1), 1 (2012). Crossref

13. A. B. Harris, A. Aharony, O. Entin-Wohlman. J. Phys.: Condens. Matter. 20, 434202 (2008).

14. A. R. Yuldasheva, N.M. Nugaeva. Letters on Materials. 9 (3), 354 (2019). (in Russian) [А. Р. Юлдашева, Н. М. Нугаева. Письма о материалах. 9 (3), 354 (2019).] Crossref

15. I.F. Sharafullin, N.M. Nugaeva, M.Kh. Kharrasov. Letters on Materials. 9 (4), 499 (2019). Crossref

16. I. F. Sharafullin, M. Kh. Kharrasov, H. T. Diep. Phys. Rev. B. 99, 214420 (2019). Crossref

17. S. E. Hog, H. T. Diep. Journal of Magnetism and Magnetic Materials. 400, 276 (2016). $\underline{\text { Crossref }}$

18. N. N. Bogolyubov, S. V. Tyablikov. Soviet Physics Doklady. 4, 589 (1959). 